TITLE:

\title{
Contribution of convection to spatiotemporal stripe patterns formed by Ag and Sb coelectrodeposition.
}

$\operatorname{AUTHOR}(\mathrm{S})$ :

Nagamine, Yuko; Yoshikawa, Kenichi

CITATION:

Nagamine, Yuko ...[et al]. Contribution of convection to spatiotemporal stripe patterns formed by Ag and Sb coelectrodeposition.. Chaos 2010, 20 (2): 023117.

ISSUE DATE:

2010-06

URL:

http://hdl.handle.net/2433/126665

RIGHT:

(C) 2010 American Institute of Physics 


\title{
Contribution of convection to spatiotemporal stripe patterns formed by $\mathrm{Ag}$ and $\mathrm{Sb}$ coelectrodeposition
}

\author{
Yuko Nagamine ${ }^{1, a)}$ and Kenichi Yoshikawa ${ }^{1,2}$ \\ ${ }^{1}$ Spatio-Temporal Order Project, ICORP, JST, Kyoto 606-8502, Japan \\ ${ }^{2}$ Department of Physics, Graduate School of Science, Kyoto University, Kyoto 606-8502, Japan
}

(Received 16 June 2009; accepted 5 April 2010; published online 17 June 2010)

\begin{abstract}
Various spatiotemporal patterns of dark and light stripes are formed on the surface of an electrode put in an electrolyte solution in a $\mathrm{Ag}$ and $\mathrm{Sb}$ coelectrodeposition system. In this study, we investigate the effect of natural convection of the solution on these spatiotemporal patterns. When the electrode is placed vertically, natural convection generally emerges in the electrolyte solution in the vicinity of the electrode surface during electrodeposition and flows upward along the electrode surface. When convective flow along the electrode surface was eliminated by placing the electrode horizontally, the configuration of the one-directional traveling waves (anisotropic shape) changed to an isotropic shape. This indicates that the formation of an anisotropic shape for one-directional traveling waves is due to upward convective flow along the electrode surface. (C) 2010 American Institute of Physics. [doi:10.1063/1.3413962]
\end{abstract}

Spatiotemporal patterns are formed in nonequilibrium systems that are constantly supplied with energy and/or materials. Spatiotemporal patterns are also formed in a $\mathrm{Ag}$ and $\mathrm{Sb}$ coelectrodeposition system. In this system, various spatiotemporal patterns of dark and light stripes emerge on the electrode surface in the electrolyte solution, and these patterns are formed by metals (Ag and Sb). However, the mechanism of pattern formation has not yet been determined. To clarify this mechanism, it is important that we understand the effect of natural convection of the solution on the spatiotemporal patterns. In this study, we compare the shape of the pattern under convection along the electrode surface to that without convection by changing the orientation of the electrode. As a result, configurations with an anisotropic shape that emerged under convection along the electrode surface changed to show an isotropic shape in the absence of convection. This indicates that the shape of the pattern is influenced by convection. In addition, the mechanism of pattern emergence is not related to convection because the pattern emerged even in the absence of convection.

\section{INTRODUCTION}

Spatiotemporal pattern formation is a representative characteristic of nonequilibrium systems. Recently, aspects of materials science concerning spatiotemporal phenomena in nonequilibrium systems, ${ }^{1-4}$ exemplified by the BelousovZhabotinsky (BZ) reaction, ${ }^{1}$ have attracted considerable attention; for example, hydrodynamic patterns, ${ }^{5}$ defect patterns in liquid crystals, ${ }^{6}$ adsorbate patterns, ${ }^{7-9}$ and morphological patterns of polymer blends ${ }^{10}$ have been observed. In addi-

\footnotetext{
${ }^{\text {a) }}$ Author to whom correspondence should be addressed. Present address: Yoshikawa Laboratory, Department of Physics, Graduate School of Science, Kyoto University, Oiwakecho Kitashirakawa, Sakyo-ku, Kyoto 606-8502, Japan. Telephone: +81-75-753-3671. FAX: +81-75-7533779. Electronic mail: nagamine@chem.scphys.kyoto-u.ac.jp.
}

tion, self-organized spatiotemporal patterns are often seen in biology, for instance, in neuron-network firing patterns in the brain $^{11,12}$ and in patterns in the skins of fish and mammals. ${ }^{13,14}$ The study of materials in nonequilibrium systems is very important not only so that we can gain a basic understanding of nature but also so that we can apply the characteristics of biological systems to practical engineering including brain-computing, such as analog-computing, ${ }^{15}$ and brain-learning functions. ${ }^{11,16}$

The $\mathrm{Ag}$ and $\mathrm{Sb}$ coelectrodeposition system, in which $\mathrm{Ag}$ and $\mathrm{Sb}$ atoms consistently accumulate on an electrode surface from a solution during electrodeposition, ${ }^{17-21}$ also exhibits spatiotemporal behavior in a nonequilibrium system. In this system, various spatiotemporal patterns are formed on the electrode surface during electrodeposition, depending on the experimental conditions; e.g., a spiral pattern, a target pattern, a spot pattern, or a wave pattern, all of which consist of light and dark regions. Intriguingly and uniquely, the spatiotemporal patterns in this system are formed by metals. The light and dark regions of the pattern in the $\mathrm{Ag}$ and $\mathrm{Sb}$ coelectrodeposition system have been reported to be rich in $\mathrm{Ag}$ and $\mathrm{Sb}$, respectively. ${ }^{19}$ However, the mechanism that underlies the emergence of these patterns formed by hard materials has not yet been adequately clarified.

The authors have investigated the mechanism of pattern formation for three kinds of traveling stripe patterns [one pattern exhibits an isotropic shape and the other two show one-directional anisotropic shapes (Fig. 1)]. They all appear in the same phase diagram versus the current density applied to an electrode. ${ }^{18}$ In our previous report concerning the time evolution of stripe width, ${ }^{20,21}$ the phase separation of $\mathrm{Ag}$ and $\mathrm{Sb}$ in the electrodeposition system was suggested as a plausible mechanism for the formation of the former pattern with an isotropic shape, ${ }^{20}$ while a reaction-diffusion system with convective flow has been proposed to explain the pattern formation for the two other patterns with one-directional an- 
(a) complex labyrinthine structure

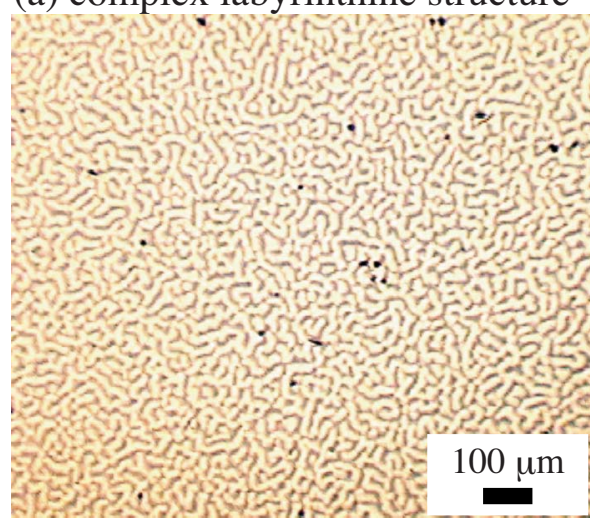

\section{(b) small wave structure}

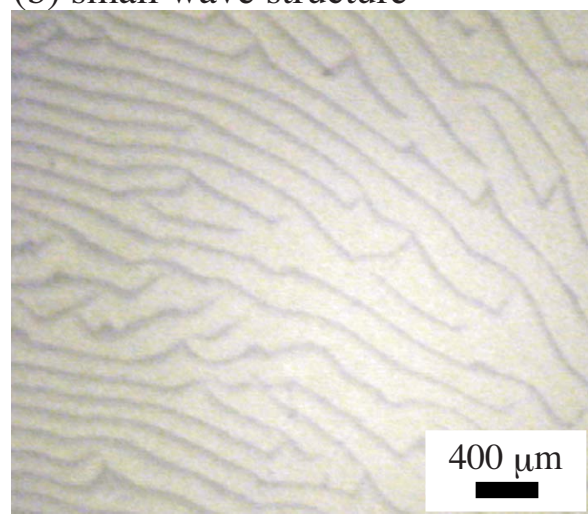

\section{(c) big wave structure}



FIG. 1. (Color online) Optical microscope images of three kinds of traveling stripe patterns in the vertical electrode condition.

isotropic shapes. ${ }^{21}$ Convection generally emerges in the vicinity of the electrode surface in the electrolyte solution during electrodeposition. In an electrodeposition system, the density of the solution near an electrode is different from that in the bulk solution because metal ions in the solution in the vicinity of an electrode surface are consumed by adsorption to the electrode surface during electrodeposition. Therefore, the buoyancy effect leads to an upward flow of the solution along the electrode surface, which is called natural convection. ${ }^{22,23}$

In this study, to investigate the contribution of convective flow ${ }^{24,25}$ to the formation of the latter two patterns with one-directional anisotropic shapes, natural convective flow along the electrode surface was eliminated. Under this condition, pattern formation on the electrode surface was observed.

\section{EXPERIMENTAL}

As a working electrode on which $\mathrm{Ag}$ and $\mathrm{Sb}$ were electrodeposited, a thin film of $\mathrm{Au}(111)$ deposited on one side of an insulating material, mica $\left(1 \times 2 \mathrm{~cm}^{2}\right)$, was used. On this $\mathrm{Au}(111)$ surface upon which $\mathrm{Ag}$ and $\mathrm{Sb}$ are electrodeposited, spatiotemporal patterns appear. The counterelectrode was a $2 \times 3 \mathrm{~cm}^{2} \mathrm{Ag}$ plate, a central part $\left(1 \times 2 \mathrm{~cm}^{2}\right)$ of which was removed so that the pattern emerging on the $\mathrm{Au}(111)$ surface of the working electrode could be viewed from outside the glass container (Fig. 2). During electrodeposition, the patterns on the working electrode were observed through an optical microscope with a zoom lens. The distance between the working electrode and the counterelectrode was $1 \mathrm{~cm}$.

In our usual experimental setup, the electrodes are suspended in the electrolyte solution vertically [setup A in Fig. 2(a)]. In this condition, natural convective flow proceeds upward along the electrode surface. To eliminate this natural upward convection, in this study the electrodes were placed horizontally [setups B and C in Figs. 2(b) and 2(c)]. To place the electrodes horizontally, a support stage for the electrodes (Fig. 3) constructed of transparent acrylic plates was placed in the container. In the completed support stage, two parallel square acrylic plates $\left(6.5 \times 6.5 \times 0.1 \mathrm{~cm}^{3}\right)$ sandwich four cubic acrylic spacers $\left(1 \times 1 \times 1 \mathrm{~cm}^{3}\right)$ at all four corners to maintain a distance of $1 \mathrm{~cm}$ between the square plates. The two square plates sandwiching spacers were then inserted in the foothold.

In this study, two horizontal electrode setups were used [setups B and C in Figs. 2(b) and 2(c)]. In the horizontal electrode setup B, the working electrode lies on the upper surface of the upper acrylic plate (area A in Fig. 3). In this setup, the $\mathrm{Au}(111)$ surface faces upward. The change in the appearance of the electrodeposited $\mathrm{Au}(111)$ surface was recorded from above using a video camera and an optical microscope after the electrodeposition of Ag and Sb started. In the horizontal electrode setup C, the working electrode lies on the under surface of the upper acrylic plate (area B in Fig. 3). In this setup, the $A u(111)$ surface faces downward. In this case, the $\mathrm{Au}(111)$ surface was observed from the bottom of the container during electrodeposition. In both horizontal working electrode conditions, the counterelectrode was fixed to the upper surface of the lower acrylic plate (area $\mathrm{C}$ in Fig. 3). In the horizontal setup B, convective flow would be expected to occur perpendicular to the electrode surface because convection is due to the buoyancy effect of gravity and convection would principally occur upward [Fig. 2(b)]. Initially, to investigate the influence of the convection flow perpendicular to the electrode surface on pattern shape, a setup in which the electrodes are oriented $90^{\circ}$ relative to those in setup A was used instead of setup B. The direction of orientation is opposite to that in setup C. In this setup, the working electrode was placed below the counterelectrode, and the potential line between the working and counterelectrodes is 
(a)

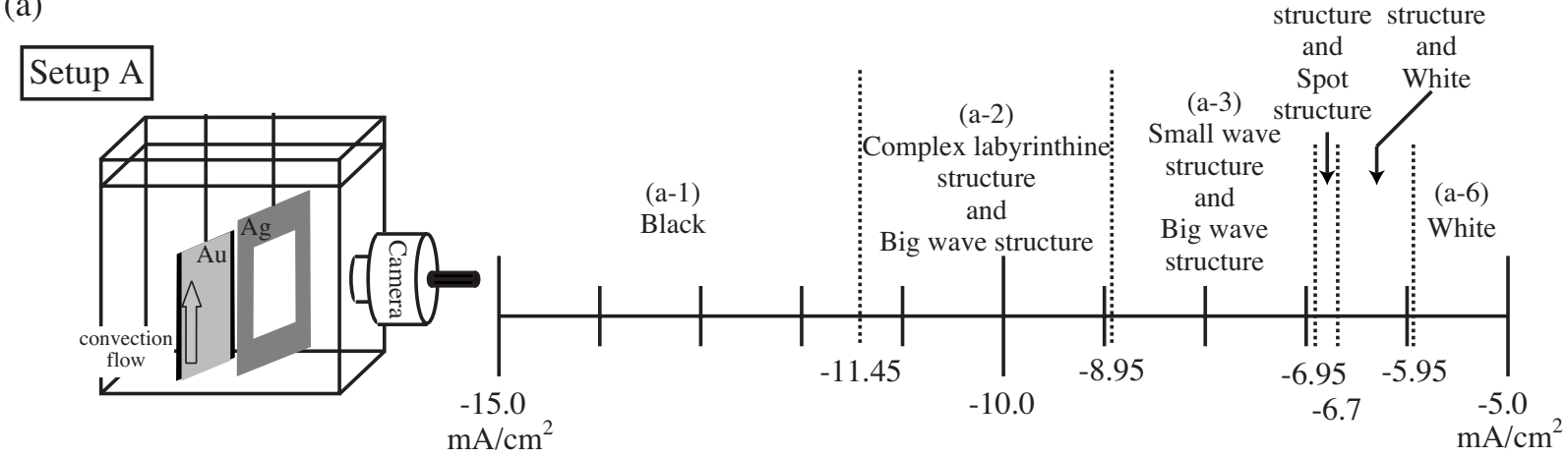

(b)

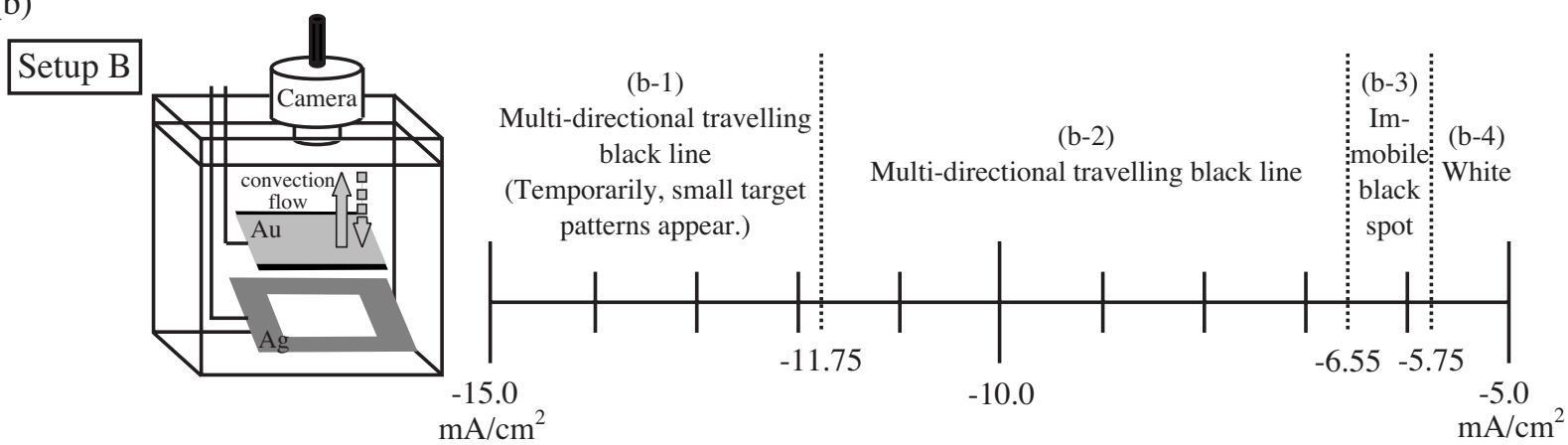

(c)
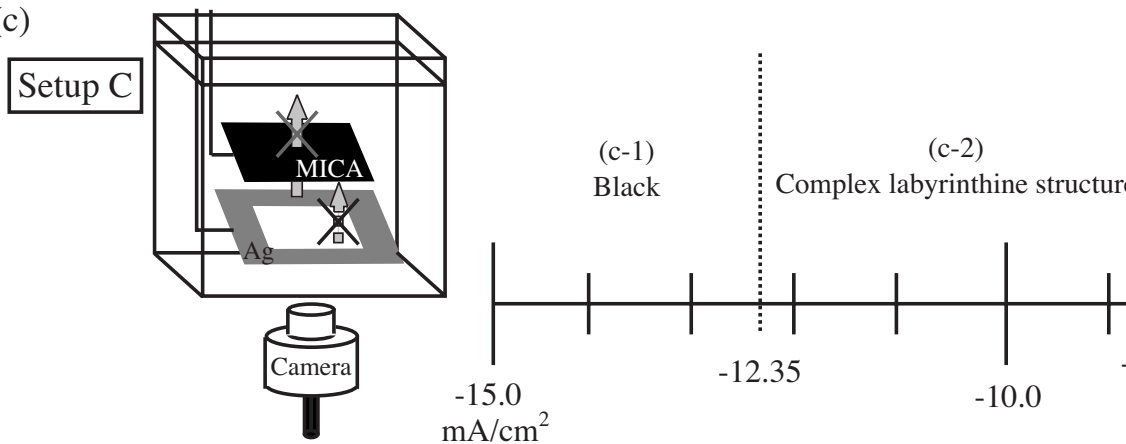

(c-4) (c-5)

$\mathrm{mA} / \mathrm{cm}^{2}$

FIG. 2. Phase diagrams of spatiotemporal patterns appearing on the electrode surface for the three electrode setups. (a) Setup A: vertical electrode setup. (b) Setup B: horizontal electrode setup in which the working electrode is located above the counterelectrode and the Au(111) surface faces upward. (c) Setup C: horizontal electrode setup in which the working electrode is located above the counterelectrode and the Au(111) surface faces downward. The gray solid arrows indicate the direction of natural convective flow. The gray dotted arrows in (b) and (c) represent the flow of the bulk electrolyte solution, following natural convection.

more parallel than that in setup B. However, the concentrated Ag solution, which develops from the surface of the upper $\mathrm{Ag}$ counterelectrode into the solution during electrodeposition, descended directly to the lower working electrode surface. The descending concentrated Ag solution could be recognized by a visual difference in the refractive index of the solution through the eye observation. The concentration of the solution near the working electrode was greatly altered by the descending concentrated Ag solution. In fact, the electrodeposited working electrode surface became whitish (Ag color) and the spatiotemporal pattern did not appear on the electrode surface. Therefore, the results for the setup in which the electrode is oriented $90^{\circ}$ from that in setup A are not shown in this study. The positions of the working and counterelectrodes were exchanged, and setup B was examined to investigate the effect of convection perpendicular to



FIG. 3. The support stage used to fix the electrode horizontally. (a) Upper surface of the topmost square acrylic plate. (b) Under surface of the topmost square acrylic plate. (c) Upper surface of the lower acrylic plate. 
the electrode surface on the pattern shape. Convection perpendicular to the electrode surface in setup B was confirmed through the observation of the microglass beads added in the solution by an optical microscope. ${ }^{26}$ On the other hand, in the horizontal setup $\mathrm{C}$, upward natural convection emerging on the electrode surface due to the buoyancy effect is blocked by the electrode body, and the solution cannot flow [Fig. 2(c)]. Therefore, in setup C, convective flow does not occur near the electrode surface. (The absence of convection in setup $\mathrm{C}$ was also checked by lateral observation with an optical microscope. ${ }^{26}$ )

To prepare the electrolyte solution used for the coelectrodeposition of $\mathrm{Ag}$ and $\mathrm{Sb}$, an aqueous solution including $0.26 M \quad \mathrm{AgNO}_{3}, \quad 0.27 M \quad \mathrm{~K}_{4} \mathrm{Fe}(\mathrm{CN})_{6} \cdot 3 \mathrm{H}_{2} \mathrm{O}$, and $0.27 M$ $\mathrm{K}_{2} \mathrm{CO}_{3}$ in distilled water was boiled. After the solution was cooled, the concentration for $\mathrm{Ag}$ was titrated. To the $0.15 \mathrm{M}$ $\mathrm{Ag}$ aqueous solution, $0.036 \mathrm{M} \mathrm{KSbOC}_{4} \mathrm{H}_{4} \mathrm{O}_{6} \cdot 3 \mathrm{H}_{2} \mathrm{O}, 1.5 \mathrm{M}$ $\mathrm{KSCN}$, and $0.21 M \mathrm{KNaC}_{4} \mathrm{H}_{4} \mathrm{O}_{6} \cdot 4 \mathrm{H}_{2} \mathrm{O}$ were added. With an electrolyte solution of this composition, a complex labyrinthine structure, a small wave structure, and a big wave structure appear on the surface of the working electrode during electrodeposition within their respective ranges of current density applied to the electrode in the constant-current mode [to maintain a constant current, the potentiostat-galvanostat (HA-151, Hokuto Denko, Tokyo, Japan) was used in galvanostat mode], as shown in the phase diagram in our previous paper, ${ }^{18}$ where the electrodes were suspended vertically in the electrolyte solution. In this study, the coelectrodeposition of $\mathrm{Ag}$ and $\mathrm{Sb}$ was carried out at room temperature and within the same range of current density but with the electrodes in a horizontal orientation, and the patterns that appeared on the $\mathrm{Au}(111)$ surface of the working electrode were observed.

\section{A. Surface element analysis of the pattern surface}

After the spatiotemporal patterns were observed, the applied current was turned off. Without the applied current, the spatiotemporal patterns on the working electrode instantly stabilized. The working electrode was removed from the electrolyte solution and dried in air after being rinsed with pure water. An element analysis was performed for the dry pattern surfaces on the working electrode using an energy dispersive x-ray spectrometer (EDX) (JSM-5600, JEOL, Tokyo, Japan), which can disperse the energy of characteristic $\mathrm{x}$ rays emitted from a sample after incidence of the electron beam. Through the dispersion of $x$ rays, the energy spectra of the emitted characteristic $\mathrm{x}$-ray can be obtained. These spectra include intensity peaks due to elements in the measurement area of the sample. The intensities of the peaks in the detected spectra can finally be converted to the ratios of the atomic percentages of the respective elements. To obtain the average concentrations for patterns that included dark and light stripes, measurements were made over an area wider than the pattern size (the actual measurement area was from $1.5 \times 1.1$ to $2.5 \times 1.9 \mathrm{~mm}^{2}$ and was adjusted depending on the pattern size). To convert the intensities of characteristic x-ray peaks to the relative atomic percentages of the elements, well-known empirical calibration factors of stan- dard samples for the respective elements were used. ${ }^{27}$ The electron beam intensity used in this study was $20.0 \mathrm{kV}$.

\section{B. Potential measurement for the working electrode}

As patterns appeared on the working electrode surface, the time evolution of the potential of the working electrode was examined against the $\mathrm{Ag} / \mathrm{AgCl}$ reference electrode. The reference electrode was placed near the working electrode. The potential value varied after the beginning of electrodeposition and initially showed a rapid decrease with time. Therefore, stable potential values were taken after a long time had passed. After the potential was measured, the potential values against the $\mathrm{Ag} / \mathrm{AgCl}$ reference electrode were recalculated to give values versus standard hydrogen electrode (SHE).

\section{RESULTS}

Figure 2(a) shows a phase diagram of the patterns versus the current density applied to the electrode in the vertical electrode setup (setup A). In the vertical electrode setup, both types of traveling stripe patterns were observed, i.e., an isotropic shape [complex labyrinthine structure with a size of $\sim 10 \mu \mathrm{m}$ and a wave speed of $\sim 1 \mu \mathrm{m} / \mathrm{s}$, Fig. 1(a) $]$ and anisotropic shapes [a small wave structure with a stripe width of $\sim 100 \mu \mathrm{m}$ and a wave speed of $\sim 10 \mu \mathrm{m} / \mathrm{s}$, and a big wave structure with a stripe width of $\sim 1 \mathrm{~mm}$ and a wave speed of $\sim 100 \mu \mathrm{m} / \mathrm{s}$, Figs. 1(b) and 1(c); these two latter patterns are one-directional traveling stripe patterns], depending on the range of the current density applied to the electrode (pattern formation in the vertical electrode condition was discussed in our previous paper ${ }^{18}$ ).

Figure 2(a) shows a phase diagram of the spatiotemporal patterns in the vertical electrode setup (setup A). Under a low current value $\left(\mathrm{I}<-11.45 \mathrm{~mA} / \mathrm{cm}^{2}\right.$, where $\mathrm{I}$ is the current density), the electrode surface becomes uniformly black [phase (a-1)] and, if the current value becomes higher $\left(-11.45 \mathrm{~mA} / \mathrm{cm}^{2}<\mathrm{I}<-8.95 \mathrm{~mA} / \mathrm{cm}^{2}\right)$, two kinds of spatiotemporal patterns, a complex labyrinthine structure and a big wave structure, appear simultaneously on the electrode surface [phase (a-2)]. If the current value increases even higher $\left(-8.95 \mathrm{~mA} / \mathrm{cm}^{2}<\mathrm{I}<-6.95 \mathrm{~mA} / \mathrm{cm}^{2}\right)$, two kinds of spatiotemporal patterns with a different combination of patterns (small wave structure and big wave structure) appear on the electrode surface [phase (a-3)] In a current range of $-6.95 \mathrm{~mA} / \mathrm{cm}^{2}<\mathrm{I}<-6.7 \mathrm{~mA} / \mathrm{cm}^{2}$, a small wave structure and a spot structure emerge on the electrode surface [phase (a-4)]. At a higher current range of $-6.7 \mathrm{~mA} / \mathrm{cm}^{2}$ $<\mathrm{I}<-5.95 \mathrm{~mA} / \mathrm{cm}^{2}$, a spot structure appears on the upper side of the electrode surface and the lower surface becomes uniformly white [phase (a-5)]. At a high current range $\left(-5.95 \mathrm{~mA} / \mathrm{cm}^{2}<\mathrm{I}\right)$, the entire electrode surface becomes white [phase (a-6)].

Within the same current density range $\left(-15.0 \mathrm{~mA} / \mathrm{cm}^{2}\right.$ $\left.<\mathrm{I}<-5.0 \mathrm{~mA} / \mathrm{cm}^{2}\right)$ as in setup A, electrodeposition on the working electrode also occurred under the two setups with horizontal electrodes (setups B and C), and the phase dia- 
(a) $50 \mathrm{~s}$

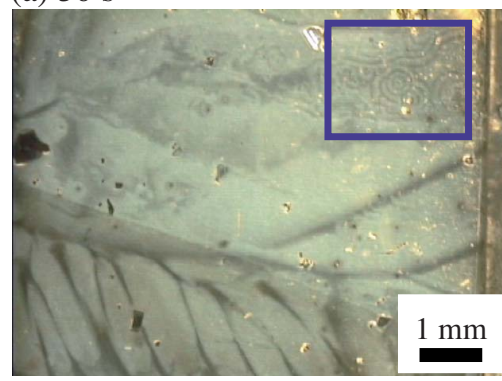

(d) $119 \mathrm{~s}$

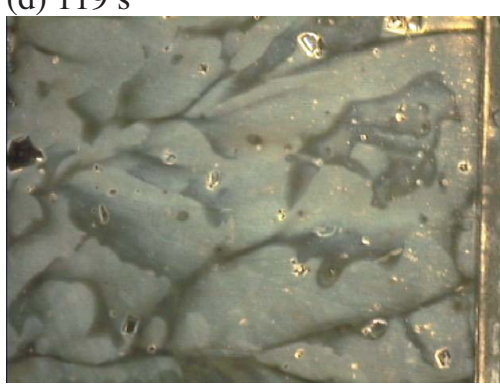

(b) $73 \mathrm{~s}$

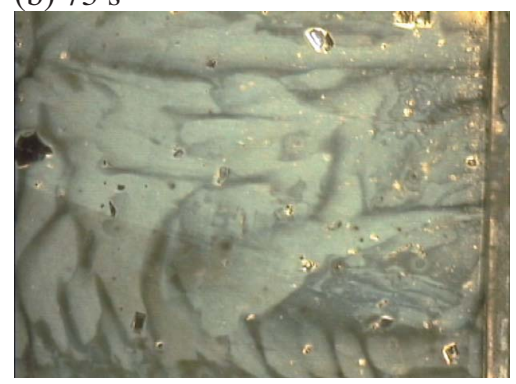

(c) $88 \mathrm{~s}$



(e) $46 \mathrm{~s}$

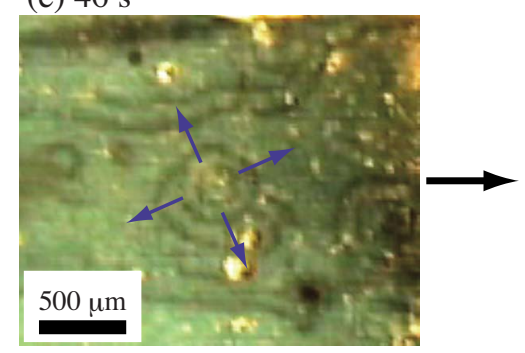

(f) $50 \mathrm{~s}$

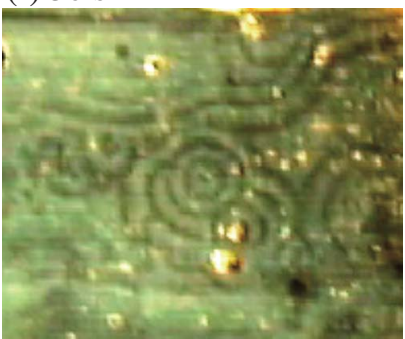

FIG. 4. (Color online) Time series of optical microscope images of the surface of the working electrode in phase (b-1) in Fig. 2(b). (e) and (f) show time-series expanded images of the area surrounded by the rectangle in (a), where small propagating target patterns emerge. Time lapses in (a)-(f) start from the onset of $\mathrm{Ag}$ and $\mathrm{Sb}$ coelectrodeposition (enhanced online).[URL: http://dx.doi.org/10.1063/1.3413962.1]

grams of the spatiotemporal patterns that appeared on the surface of the working electrode were obtained [Figs. 2(b) and $2(\mathrm{c})]$.

In setup $B$, under a low current value $\left(\mathrm{I}<-11.75 \mathrm{~mA} / \mathrm{cm}^{2}\right)$, multidirectional traveling black lines appear on the electrode surface. Isotropic small target patterns appear temporarily in the interspace of the multidirectional traveling black lines [phase (b-1)].

Figure 4 shows optical microscope images of the working electrode surface in phase (b-1). In that condition, traveling black lines appear. These lines appear to travel in a variety of directions. The appearing lines do not travel in a same specific direction. The distance between the lines is about $1 \mathrm{~mm}$ and they travel at a speed of about $80 \mu \mathrm{m} / \mathrm{s}$. At the initial stage of electrodeposition, small propagating target patterns appear together with these black lines [Figs. 4(a), 4(e), and 4(f)]. The stripe wavelength of the target pattern is about $100 \mu \mathrm{m}$ and the stripes travel at a speed of about $30 \mu \mathrm{m} / \mathrm{s}$. The target patterns survive on the electrode surface for about $40 \mathrm{~s}$ from when they emerge and are eventually eliminated by the traveling black lines [Figs. 4(b) and 4(c)]. After the target patterns disappear, the black lines cover the entire electrode surface [Fig. 4(d)]. The distances between stripes for the multidirectional traveling black lines and the small target patterns are very close to those for the big and small wave structures of the one-directional traveling patterns with anisotropic shapes, which emerge in setup A, respectively. Thus, these isotropic patterns (multidirectional traveling black lines and small target patterns) might be derived from big and small wave structures, respectively. However, while the speed at which stripes travel for the small target patterns is similar to that for the small wave structure, the multidirectional traveling black lines move more slowly than the big wave structure. This result regarding velocities reveals that the effect of convection on the pattern might vary according to the kind of pattern.

At a higher current range of $-11.75 \mathrm{~mA} / \mathrm{cm}^{2}<\mathrm{I}$ $<-6.55 \mathrm{~mA} / \mathrm{cm}^{2}$, only the multidirectional traveling black lines emerge [phase (b-2)]. The small target pattern does not emerge, unlike in phase (b-1). In the even higher current range of $-6.55 \mathrm{~mA} / \mathrm{cm}^{2}<\mathrm{I}<-5.75 \mathrm{~mA} / \mathrm{cm}^{2}$, immobile black spots appear [phase (b-3)]. In the high current range $\left(-5.75 \mathrm{~mA} / \mathrm{cm}^{2}<\mathrm{I}\right)$, the entire electrode surface becomes white [phase (b-4)].

In setup $\mathrm{C}$, under a low current value $(\mathrm{I}<-12.35$ $\mathrm{mA} / \mathrm{cm}^{2}$ ), the electrode surface becomes black [phase (c-1)]. At a higher current range of $-12.35 \mathrm{~mA} / \mathrm{cm}^{2}<\mathrm{I}$ $<-8.55 \mathrm{~mA} / \mathrm{cm}^{2}$, the complex labyrinthine structure appears [phase $(c-2)]$. In the even higher current range of $-8.55 \mathrm{~mA} / \mathrm{cm}^{2}<\mathrm{I}<-5.7 \mathrm{~mA} / \mathrm{cm}^{2}$, isotropic traveling patterns of two different sizes (small target pattern and big target pattern) emerge on the electrode surface [phase (c-3)].

Figures 5 and 6 show the small and big target patterns in phase (c-3), respectively. The small target patterns shown in Fig. 5(a) start to appear soon after the start of the electrodeposition of $\mathrm{Ag}$ and $\mathrm{Sb}$. The stripe wavelength in the small target pattern is about $100 \mu \mathrm{m}$ and the wave velocity is about $30 \mu \mathrm{m} / \mathrm{s}$. The small target patterns appear for about $75 \mathrm{~s}$. Thereafter, the small target patterns are eliminated by bold black curved lines that travel in a variety of directions [Figs. 5(b) and 5(c)]. Eventually, the bold black curved lines cover the entire electrode surface [Fig. 5(d)]. The shape of these curved lines over a suitable time is shown in Fig. 6. The bold black curves finally form the big target pattern shape. The stripe wavelength in the big target pattern is around $1 \mathrm{~mm}$ and the stripe velocity is about $30 \mu \mathrm{m} / \mathrm{s}$. These stripe wavelengths for the small and big target patterns 

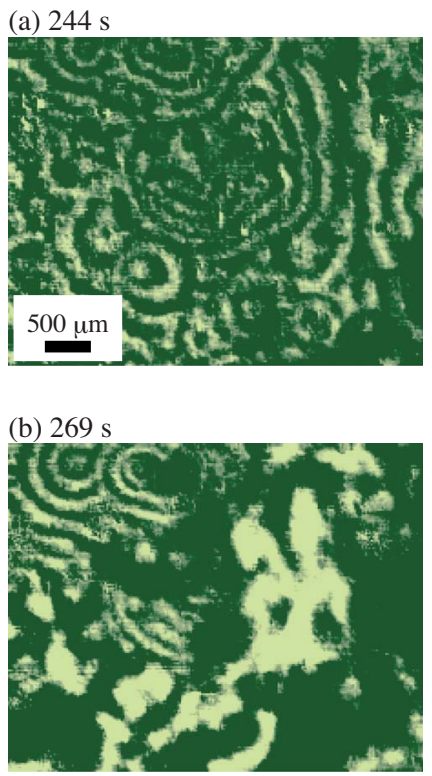

(c) $319 \mathrm{~s}$

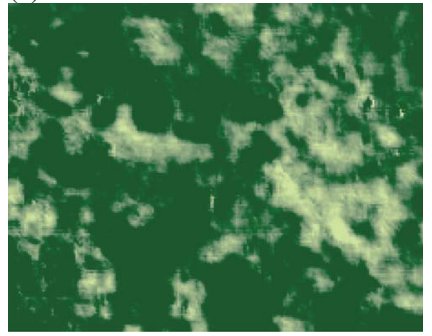

(d) $419 \mathrm{~s}$

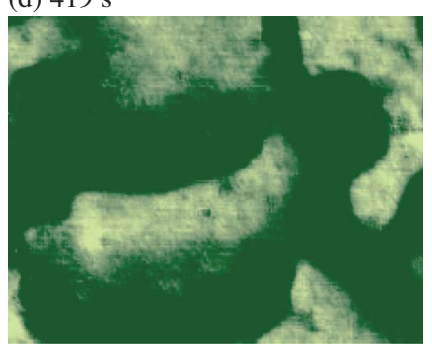

FIG. 5. (Color online) Time series of optical microscope images of the surface of the working electrode in phase (c-3) in Fig. 2(c) at an early stage after the start of electrodeposition. In (a)-(d), images that have been time averaged over the pattern periods are subtracted from the respective raw grayscale images to enhance the image contrast of the small target pattern. The images are shown in pseudocolor. Time lapses in (a)-(d) start from the onset of $\mathrm{Ag}$ and $\mathrm{Sb}$ coelectrodeposition (enhanced online).[URL: http://dx.doi.org/10.1063/1.3413962.2]

are also very similar to those of the small and big wave structures of one-directional traveling patterns with anisotropic shapes, respectively, which emerge in the vertical setup A. Therefore, these isotropic target patterns might also be derived from the small and big wave structures, respectively. However, while the stripe velocity for the small target pattern is close to that for the small wave structure, the stripe velocity for the big target pattern is much slower than that for the big wave structure, as well as those for the small target patterns and multidirectional traveling black lines in phase (b-1).
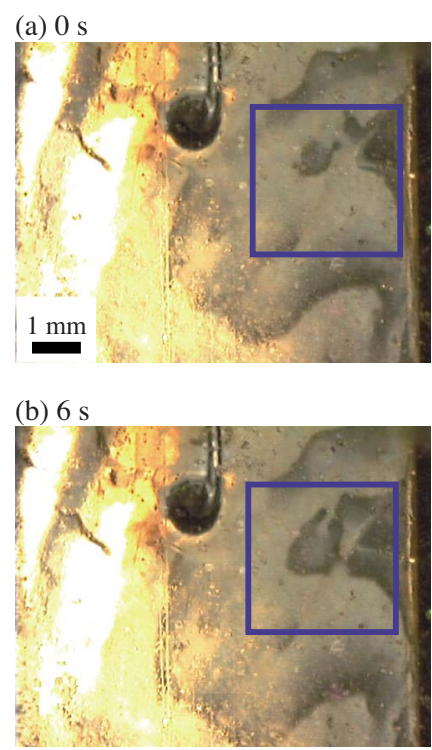

(c) $28 \mathrm{~s}$

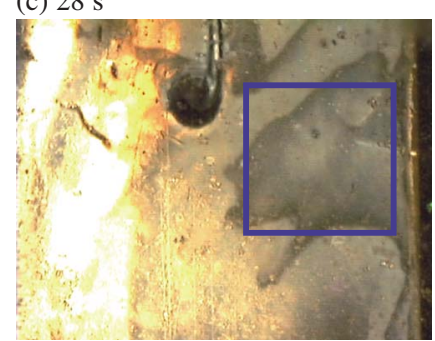

(d) $42 \mathrm{~s}$

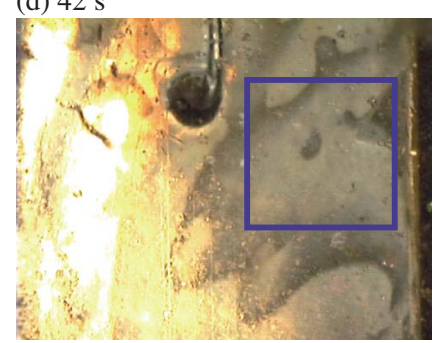

(e) $56 \mathrm{~s}$

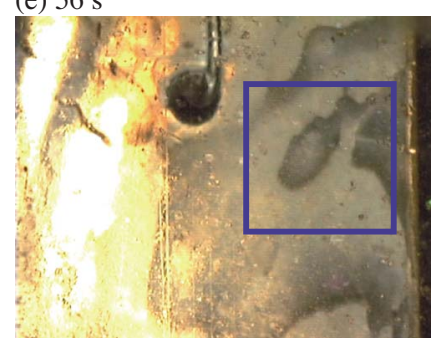

FIG. 6. (Color online) Time series of optical microscope images of the surface of the working electrode in phase (c-3) in Fig. 2(c) at $11 \mathrm{~m}, 30 \mathrm{~s}$ after the start of electrodeposition. The rectangle encloses the area where the big target pattern is emerging. The two subsequent target patterns are shown Time lapses in (b)-(e) are relative to (a) (enhanced online).[URL: http://dx.doi.org/10.1063/1.3413962.3]

At a current range of $-5.7 \mathrm{~mA} / \mathrm{cm}^{2}<\mathrm{I}$ $<-5.35 \mathrm{~mA} / \mathrm{cm}^{2}$, the small target patterns appear [phase (c-4)]. The small target patterns cover the entire electrode surface in this phase. At a high current range $\left(-5.35 \mathrm{~mA} / \mathrm{cm}^{2}<\mathrm{I}\right)$, the entire electrode surface becomes white [phase (c-5)]. In the boundary between the individual 
phases in Fig. 2, the pattern shape does not change gradually from one kind of pattern to another but rather changes suddenly.

As a result, in the horizontal electrode setup B, neither of the three traveling stripe patterns in the vertical electrode setup A (complex labyrinthine structure, small wave structure, and big wave structure) emerges on the electrode surface. At a current density range lower than $-11.75 \mathrm{~mA} / \mathrm{cm}^{2}$, only a stripe pattern similar to the small wave structure is observed [this pattern is called the small target pattern in phase (b-1)]. However, this pattern has an isotropic shape, unlike the original small wave structure of the anisotropic one-directional traveling pattern.

In contrast, in the horizontal electrode setup C, among the three traveling stripe patterns that appear in the vertical electrode setup A, only a complex labyrinthine structure with an isotropic shape survives on the electrode surface [phase (c-2)]. However, two patterns similar to the small and big wave structures, respectively, are observed [these similar patterns are called the small and big target patterns in phases (c-3) and (c-4)]. The shapes of these patterns are also isotropic, unlike the original small and big wave structures.

Among the three traveling stripe patterns that appear in the vertical electrode setup A, the complex labyrinthine structure that represents an isotropic shape could survive in the horizontal electrode setup (setup C) and the small and big wave structures that show anisotropic shapes due to onedirectional traveling waves do not appear in either of the horizontal setups (setups B and C), while similar patterns with isotropic shapes are present. These results show that the small and big wave structures emerge only in the vertical electrode setup (setup A).

Next, to determine the reason for the differences in the phase diagrams among the three setups (setups A-C), an element analysis was carried out using EDX for the pattern surfaces on the working electrode after we observed the traveling patterns. Figure 7 shows the ratios of the Ag atomic percentage to the $\mathrm{Sb}$ atomic percentage for the pattern surfaces, which are plotted against the current density applied to the electrode surface for electrodeposition. The results reveal that the ratios substantially increase at a certain value of the applied current density (critical current density: $I_{c}$ ) from a bifurcated lower value to a higher value. The lower ratio of $\mathrm{Ag}$ to $\mathrm{Sb}$ is about 5 and the higher ratio is about 25 . The critical current density values differ according to the electrode setup.

The filled dots in Fig. 7 show the ratios of $\mathrm{Ag}$ to $\mathrm{Sb}$ for the pattern surface on the working electrode samples created under setup B. The ratio of $\mathrm{Ag}$ to $\mathrm{Sb}$ in phase (b-1) is near 8 . However, in phase (b-2), the ratio of Ag to Sb suddenly increases to around $25\left(\mathrm{I}_{\mathrm{c}}{ }^{(\mathrm{B})}=-11.45 \mathrm{~mA} / \mathrm{cm}^{2}\right)$. In phases (b-3) and (b-4), this high ratio is maintained. The difference between (b-1) and (b-2) with regard to the kind of pattern is the presence or absence of the small target pattern. In phase (b-1), a pattern that shows stripe structures (small target pattern) temporarily emerges in the interspaces of the area for the multidirectional traveling black lines. On the other hand, in phase (b-2), no patterns with stripe structures similar to the small target pattern appear on the electrode surface. In
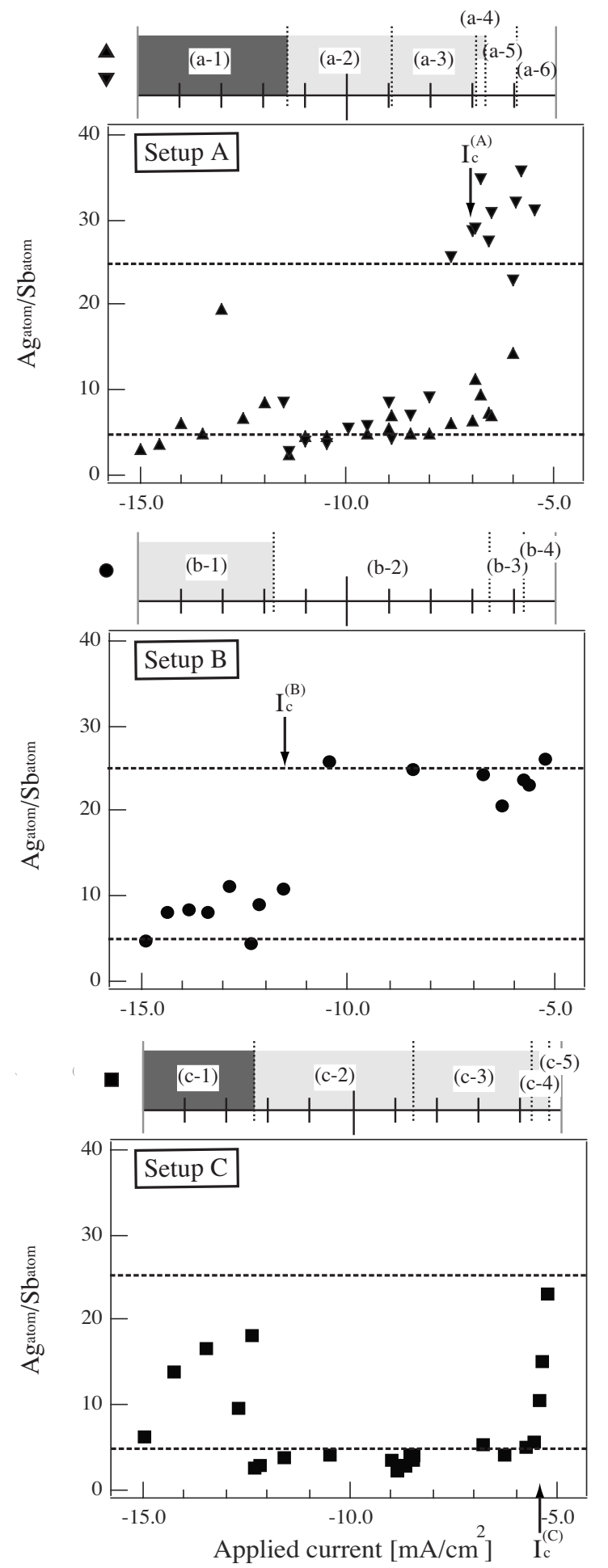

FIG. 7. Ratio of the Ag atomic percentage to the Sb atomic percentage for the electrode surface. $\boldsymbol{\Lambda}$ and $\boldsymbol{\nabla}$ : the values for the upper and lower areas of the working electrode samples created under the vertical electrode setup A, respectively. When the electrode surface is uniform in the uniformly black and white phases, only the results of element analysis for the lower or upper part are shown. : the values for the working electrode samples under the horizontal electrode setup B. $\mathbf{\square}$ : the values for the working electrode samples under the horizontal electrode setup $\mathrm{C}$. The dotted lines show the bifurcated values for the ratio of $\mathrm{Ag}$ to $\mathrm{Sb}$, i.e., 5 and 25. The phase diagrams of Fig. 2 are shown above the graph of the ratios of $\mathrm{Ag}$ to $\mathrm{Sb}$. In the phase diagram, the dark gray and light gray areas indicate the uniformly black phases and the phases where the patterns with stripe structures appear, respectively. 
phases (b-3) and (b-4), again, no patterns with stripe structures appear. These findings suggest that the large difference in the ratio of $\mathrm{Ag}$ to $\mathrm{Sb}$ between (b-1) and the other phases [(b-2), (b-3), and (b-4)] is associated with the emergence or nonemergence of patterns representing stripe structures.

The filled squares in Fig. 7 show the ratios of $\mathrm{Ag}$ to $\mathrm{Sb}$ for pattern surfaces on working electrode samples created under setup C. In the uniformly black phase (c-1), the ratios of $\mathrm{Ag}$ to $\mathrm{Sb}$ are between 5 and 20. In phase (c-2), the ratios of $\mathrm{Ag}$ to $\mathrm{Sb}$ suddenly decrease to the bifurcated lower value of about 5 , and in phases (c-3) and (c-4), the ratios remain at this low value. Furthermore, when the applied current density shifts to a more positive value, the working electrode surface becomes uniformly white (c-5) and the electrode surface shows no pattern. In this phase, the ratio of $\mathrm{Ag}$ to $\mathrm{Sb}$ substantially increases $\left(\mathrm{I}_{\mathrm{c}}{ }^{(\mathrm{C})}=-5.35 \mathrm{~mA} / \mathrm{cm}^{2}\right)$. These results mean that the ratio of $\mathrm{Ag}$ to $\mathrm{Sb}$ is low when patterns with stripe structures emerge [i.e., when a complex labyrinthine structure, small target pattern or big target pattern appears in phase (c-2), (c-3), or (c-4)]. In setup C, patterns with stripe structures appear over a wider current density range than in setup B. Accompanying this, the value of $I_{c}$ tends to be higher than that in setup B and overall the pattern surfaces tend to be richer in Sb than those in setup B.

In the vertical electrode condition (setup A), two different kinds of patterns appeared simultaneously on the upper and lower areas of the same electrodeposited working electrode surface. ${ }^{18}$ Therefore, an element analysis was performed for the upper area $(\mathbf{\Lambda})$ and the lower area $(\boldsymbol{\nabla})$ of the working electrode surface, respectively, except for the electrode sample that showed a uniformly black phase (a-1) and uniformly white phase (a-6). The results show that, in the uniformly black phase (a-1), the ratio of $\mathrm{Ag}$ to $\mathrm{Sb}$ is also about 5. On the electrode surface areas in phases (a-2), (a-3), and (a-4), which showed patterns with stripe structures, the ratios of $\mathrm{Ag}$ to $\mathrm{Sb}$ are near the bifurcated lower value of 5 [the upper and lower areas of the working electrode in phases (a-2) and (a-3), and the upper area of the electrode in phase (a-4), showed the complex labyrinthine structure, the small wave structure or the big wave structure]. In the areas of the electrode surface in phases (a-4), (a-5), and (a-6) where the spot structures or the uniformly white surfaces emerge [the lower area of the electrode in phase (a-4) and the entire areas of phases (a-5) and (a-6)], the ratios of $\mathrm{Ag}$ to $\mathrm{Sb}$ are close to the bifurcated higher value, $25\left(\mathrm{I}_{\mathrm{c}}{ }^{(\mathrm{A})}=-7.0 \mathrm{~mA} / \mathrm{cm}^{2}\right)$.

Based on the above results of the element analysis, the ratios of $\mathrm{Ag}$ to $\mathrm{Sb}$ are close to the bifurcated lower value when patterns with stripe structures emerge on the electrode surface in all three electrode setups; i.e., patterns with stripe structures emerge under Sb-rich conditions. When the phase changes to show no stripe structures, the ratio of $\mathrm{Ag}$ to $\mathrm{Sb}$ varies transitionally and becomes the bifurcated higher value in all three electrode setups. This indicates that patterns with no stripe structures emerge under Ag-rich conditions.

Furthermore, the potentials of the working electrode to which the constant current is applied under horizontal setups $\mathrm{B}$ and $\mathrm{C}$ were investigated while patterns traveled on the working electrode surface (Fig. 8). Overall, the potentials of the electrode under horizontal setup B are higher than those



FIG. 8. The potential of the working electrode vs SHE at which patterns appear. : the working electrode under the horizontal electrode setup B. $\mathbf{\square}$ : the working electrode under the horizontal electrode setup $\mathrm{C}$. The dotted line indicates the critical potential for $\mathrm{Sb}$ reduction $(-680 \mathrm{mV})$. The phase diagrams in Figs. 2(b) and 2(c) are shown above the graph of the measured potentials. In the phase diagrams, the dark gray and light gray areas indicate the uniformly black phases and the phases where the patterns with stripe structures appear, respectively.

under horizontal Setup C. Furthermore, if we compare the phase diagrams of horizontal setups $\mathrm{B}$ and $\mathrm{C}$, which are shown above the potential graph in Fig. 8, most of the potential values are below $-680 \mathrm{mV}$ versus SHE in both setups when patterns with stripe structures appear on the working electrode surface. According to our previous investigation, ${ }^{28}$ $\mathrm{Sb}$ could be reduced on the working electrode surface in the potential range below $-680 \mathrm{mV}$, together with Ag. Therefore, this trend in the potential for patterns with stripe structures implies that the working electrode surfaces where such patterns appear become $\mathrm{Sb}$ rich. This is consistent with the trend in the above element analysis.

\section{DISCUSSION}

\section{A. Effect of convection on the configuration and emergence of the small and big wave structures}

In the phase diagram of the vertical electrode setup A, where convective flow occurs along the electrode surface [Fig. 2(a)], three traveling stripe patterns appear. Among these patterns, two are anisotropic shape patterns: small and big wave structures with one-directional traveling property. However, in the phase diagrams of the horizontal electrode setups $B$ and $C$, where convective flow occurs perpendicular to the electrode surface and where there is no such flow, respectively [Figs. 2(b) and 2(c)], there are no anisotropic shape patterns, including the small and big wave structures with one-directional traveling property. Instead, there are small and big target patterns with isotropic shapes, which are similar in size to the small and big wave structures. Figure 8 shows that the potentials at which the small and big target patterns emerge are about -620 and $-680 \mathrm{mV}$, respectively. 
[The results for phase (c-4) show around $-620 \mathrm{mV}$ as a stable potential value in a long time after electrodeposition. Small target patterns then appear over the entire electrode surface. The potential values in phase (c-3) are stable at around $-680 \mathrm{mV}$ when the big target patterns appear on the entire electrode surface.] These values are very close to the potentials at which the small and big wave structures appear, which were obtained in our previous study. ${ }^{28}$ The similarity of the pattern sizes and emergence potentials also suggests that the small and big target patterns emerge via the same mechanism as the small and big wave structures, respectively. However, the changes in shape from anisotropic shapes (small and big wave structures with one-directional traveling patterns in setup A) to isotropic shapes (small and big target patterns in setups B and C) suggest that convective flow affects the pattern shape, and convective flow along the electrode surface induced by setup A leads to the anisotropic shapes of the small and big wave structures. However, the experimental finding that the small and big target patterns emerge in the absence of convection (setup C) shows that convection is not related to the mechanism of the emergence of the small and big target patterns. Thus, the mechanisms of the emergence of the small and big wave structures also does not involve convection because these mechanisms are assumed to be the same as those for the small and big target patterns, based on the above results regarding pattern sizes and emergence potentials. Although the convection influences the shape of the above patterns, it is not involved in the mechanism of pattern emergence.

Only the complex labyrinthine structure with an isotropic shape, which is a traveling stripe pattern that emerges in the vertical setup A, also appeared in the horizontal setup (setup C, no convection). This indicates that the mechanism of the emergence of the complex labyrinthine structure also is not related to convection.

Ultimately, our results show that the mechanism of the emergence of the small and big wave structures, as well as other patterns with stripe structures (complex labyrinthine structure and small and big target patterns), does not involve convection. With regard to the mechanism of the emergence of the small and big wave structures, instead of convection, a reaction-diffusion system on the electrode surface also plays a role. (This is supported by our previous investigation of the dynamic properties of the small and big wave structures. ${ }^{21}$ ) This electrodeposition system is under a constant-current mode. Thus, the potential of the local area could deviate from the average potential value of the entire electrode. If the interface solution in the vicinity of the electrode surface reacts due to the deviated potential, a stripe is formed. The electrochemical reaction that forms the stripe includes an autocatalytic process, as expected based on the experimental observation on the appearance of the traveling waves. The big difference in stripe wavelengths between the small and the big wave structures $(\sim 100 \mu \mathrm{m}$ and $\sim 1 \mathrm{~mm}$, respectively ${ }^{18}$ ) is due to differences in the autocatalytic electrochemical reactions since the kinds of patterns and types of electrochemical reactions are basically controlled by the same physical parameter, the potential value of the working electrode, ${ }^{28,29}$ and the kinds of patterns are closely related to the types of electrochemical reactions, as mediated by the potential.

Thus, the mechanism for the emergence of the small and big target patterns also involves an autocatalytic electrochemical reaction on the electrode surface since this mechanism is the same as that for the emergence of the small and big wave structures. The two types of autocatalytic electrochemical reactions occurring simultaneously on the electrode surface contribute to the temporal coexistence of the small and big target patterns (Fig. 5).

\section{B. Original mechanism for stripe propagation of the small and big wave structures}

To elucidate the mechanism of the original stripe propagation for the small and big wave structures that emerge in setup A, where we wish to exclude the effect of convection, we must consider stripe propagation for the small and big target patterns that appear in the absence of convection in setup $\mathrm{C}$, for which the mechanism of emergence is the same as that for the small and big wave structures, as mentioned above, since the experimental results for the small and big wave structures are influenced by convective flow along the electrode surface (setup A) and this discussion is more complicated than that for the small and big target patterns.

The stripes for the small and big target patterns propagate along the electrode surface in the absence of convective flow, after the stripes are formed by autocatalytic electrochemical reactions. In the reaction-diffusion system, the velocity of stripe propagation depends on both of the diffusion constant of the reactants and the reaction constant. [The simplest example is a single-component reaction-diffusion system where the velocity of the reaction-diffusion wave is proportional to $\left(k^{*} D\right)^{1 / 2}$. Here, $k$ is the reaction constant and $D$ is the diffusion constant. $]^{30,31}$ Reports in literature give diffusion constants of adsorbed $\mathrm{Ag}$ atoms on a metal surface ${ }^{32}$ and a free-diffusion constant for $\mathrm{Ag}$ ions in an aqueous solution. ${ }^{33}$ However, the reaction constant is unclear at present because the reaction details have not been elucidated. Under this situation, it is almost impossible to compare the stripe velocity of our experimental data to a calculated value in a quantitative manner. Therefore, the factor that contributes most to the mechanism of stripe propagation is still unclear.

Our calculations based on the recorded time-series images for the electrode surface show that the frequencies $f_{\mathrm{p}}$ of the pacemakers of the target patterns that emerge in setups B and $\mathrm{C}$ (without convective flow along the electrode surface) are $0.25 \mathrm{~Hz}$ (small target pattern in setup B), $0.14 \mathrm{~Hz}$ (small target pattern in setup C), and $0.016 \mathrm{~Hz}$ (big target pattern in setup C), respectively. On the other hand, the frequencies of $f_{\mathrm{w}}$ for the waves of the target patterns are $0.25 \mathrm{~Hz}$ (small target pattern in setup B), $0.17 \mathrm{~Hz}$ (small target pattern in setup C), and $0.017 \mathrm{~Hz}$ (big target pattern in setup C). Thus, $f_{\mathrm{w}}$ is slightly greater than $f_{\mathrm{p}}$. In the $\mathrm{BZ}$ reaction system, as a diffusion-controlled model of a reaction-diffusion system, $f_{\mathrm{w}}$ is less than $f_{\mathrm{p}}$ because of the curvature effect of the target pattern. ${ }^{34}$ The difference in behavior between this system and the BZ reaction system reveals that diffusion does not pre- 
dominantly control this system and the reaction supports the propagation of the stripe pattern. The activation propagation of the autocatalytic electrochemical reaction through local coupling to neighboring sites might be involved in the mechanism of stripe propagation.

A sea shell is also a spatiotemporal pattern in a solid system. As a model for this system, the reaction-diffusion model is used, and activator and inhibitor molecules diffuse and react in the solid cell of the sea shell. ${ }^{14}$ The small and big wave structures (and the small and big target patterns) in the $\mathrm{Ag}$ and $\mathrm{Sb}$ electrodeposition system are analogous to this system. The $\mathrm{Ag}$ and $\mathrm{Sb}$ ions diffuse and react on the solid electrode surface.

\section{Effect of convection on the difference in phase diagrams between the horizontal electrode setups}

Based on the results of the element analysis and the potential measurement (Figs. 7 and 8), the contribution of convection could explain the differences in phase diagrams between the horizontal electrode setups B and C. When the electrode is fixed horizontally as in setup B, the direction of the upward convective flow induced by the buoyancy effect is perpendicular to the electrode surface. In this setup, as convective flow ascends, fresh solution could descend directly toward the electrode from the bulk solution [Fig. 2(b)]. Thus, the solution near the electrode surface is always replaced by fresh high-Ag concentration bulk solution, and high-Ag concentration solution is always supplied to the interface at the electrode surface. Since the reduction potential of $\mathrm{Ag}$ is principally higher than that of $\mathrm{Sb},{ }^{28}$ the electrode prefers to reduce $\mathrm{Ag}$ on the electrode surface rather than $\mathrm{Sb}$ under the constant-current mode. Thus, when high-Ag concentration solution is supplied to the electrode surface in setup B, Ag dominantly continues to be adsorbed on the electrode surface. As a result, the surfaces of the electrode samples created under setup B become Ag rich, and it is difficult for patterns that show stripe structures, which otherwise emerge under Sb-rich conditions, to appear.

With setup C, since upward convection due to the buoyancy effect is blocked by the electrode body itself [Fig. 2(c)], convective flow does not develop. Therefore, the solution near the electrode surface is not replaced by fresh high-Ag concentration bulk solution. In this condition, after Ag adsorption to the electrode surface occurs in preference to $\mathrm{Sb}$ adsorption due to the higher reduction potential of $\mathrm{Ag}, \mathrm{Ag}$ ions are consumed in the solution close to the electrode surface. As a result of this change in the Ag concentration of the solution near the electrode surface, the potential decreases to a value below the critical potential for $\mathrm{Sb}$ reduction, and $\mathrm{Sb}$ reduction is promoted to maintain the constant current. Therefore, the surfaces of samples created under setup C become $\mathrm{Sb}$ rich and patterns with a stripe structure tend to emerge.

Such a difference in the convection conditions contributes to the difference in the phase diagrams, and the value of $I_{c}$ changes depending on the presence or absence of patterns with stripe structures, which require an Sb-rich condition for formation.

\section{Relation between the types of patterns that show stripe structures and the ratio of $\mathrm{Ag}$ to $\mathrm{Sb}$}

Figure 7 shows that the ratios of $\mathrm{Ag}$ to $\mathrm{Sb}$ for the pattern surface on the working electrode are almost constant (about 5) in all three electrode setups when traveling patterns with stripe structures exist on the working electrode surface, regardless of the kinds of patterns that emerge. The kind of stripe pattern does not significantly depend on the ratio of $\mathrm{Ag}$ to $\mathrm{Sb}$. The kind of stripe pattern is determined by the concentrations of elements other than $\mathrm{Ag}$ and $\mathrm{Sb}$ that are also included in the pattern in small amounts such as oxygen, sulfur, potassium, and carbon (our previous study ${ }^{19}$ ). The adsorption of these elements to the electrode surface might also depend on the potential of the electrode since the kind of stripe pattern is closely associated with the potential of the electrode, ${ }^{28}$ as mentioned above.

In the uniformly black phases [(a-1) and (c-1) in Fig. 2] that appear under the vertical electrode setup A and the horizontal electrode setup $\mathrm{C}$, the ratio of $\mathrm{Ag}$ to $\mathrm{Sb}$ varies from 5 to 20. Thus, the ratio of $\mathrm{Ag}$ to $\mathrm{Sb}$ is not critically responsible for the formation of the uniformly black phase. Again, other elements also play an important role in the formation of the uniformly black phase.

The results of the element analysis in our previous study ${ }^{19}$ showed that the oxygen concentration at the electrodeposited surface differs greatly between the uniformly black phase and patterns with stripe structures. This also supports the above proposal.

\section{CONCLUSION}

When natural convective flow along the electrode surface is eliminated by placing electrodes horizontally, the anisotropic shapes of two spatiotemporal stripe patterns (small and big wave structures of one-directional traveling patterns) change to isotropic shapes. In addition, the sizes of the two patterns are scarcely changed. This indicates that convection along the electrode surface affects the shapes of the patterns but does not contribute to the original emergence of the patterns since the patterns could survive in the absence of convective flow.

The velocity of the traveling stripe in the reactiondiffusion system depends on both the diffusion constant and the reaction constant. Since the reaction constant in this system is not yet clear, we cannot provide a detailed discussion of the experimental velocity with a theoretical estimation. However, a frequency comparison between the pacemaker and wave of the target pattern shows that the reaction significantly supports stripe propagation by an autocatalytic electrochemical reaction through local coupling to a neighboring site of the interface solution along the electrode surface.

Our model for the effect of convection on the $\mathrm{Ag}$ and $\mathrm{Sb}$ concentrations of the solution near the electrode surface during electrodeposition could explain the results of an element analysis for the electrode surface and the potential for the electrodeposited electrode. Furthermore, the results regarding the ratios of $\mathrm{Ag}$ and $\mathrm{Sb}$ obtained by the element analysis reveal that while all kinds of patterns with a stripe structure 
emerge under Sb-rich conditions, the kind of stripe pattern does not significantly depend on the ratio of Ag to Sb. The kind of stripe pattern might be strongly controlled by the concentrations of elements other than $\mathrm{Ag}$ and $\mathrm{Sb}$, which are also present in the pattern.

\section{ACKNOWLEDGMENTS}

The element analysis data in this study were obtained by EDX, which is owned by Professor Yoshimura's laboratory at the Graduate School of Science, Kyoto University. We are also grateful to Professor Konstantin Agladze, iCeMS, Kyoto University, for his fruitful suggestions.

This work was supported in part by a Grant-in-Aid for Scientific Research (Y.N.) from the Ministry of Education, Culture, Sports, Science and Technology of Japan.

${ }^{1}$ A. N. Zaikin and A. M. Zhabotinsky, "Concentration wave propagation in two-dimensional liquid-phase self-oscillating system," Nature (London) 225, 535 (1970).

${ }^{2}$ C. Sachs, M. Hildebrand, S. Völkening, J. Wintterlin, and G. Ertl, "Spatiotemporal self-organization in a surface reaction: From the atomic to the mesoscopic scale," Science 293, 1635 (2001).

${ }^{3}$ D. Walgraef, Spatio-Temporal Pattern Formation (Springer, New York, 1997).

${ }^{4}$ C. Bowman and A. C. Newell, "Natural patterns and wavelets," Rev. Mod. Phys. 70, 289 (1998).

${ }^{5}$ R. Meyer-Spasche, Pattern Formation in Viscous Flows (Birkhäuser, Basel, 1999).

${ }^{6}$ J.-H. Huh, Y. Hidaka, A. G. Rossberg, and S. Kai, "Pattern formation of chevrons in the conduction regime in homeotropically aligned liquid crystals," Phys. Rev. E 61, 2769 (2000).

${ }^{7}$ Y.-J. Li, J. Oslonovitch, N. Mazouz, F. Plenge, K. Krischer, and G. Ertl, "Turing-type patterns on electrode surfaces," Science 291, 2395 (2001).

${ }^{8}$ M. Kim, M. Bertram, M. Pollmann, A. von Oertzen, A. S. Mikhailov, H. H. Rotermund, and G. Ertl, "Controlling chemical turbulence by global delayed feedback: Pattern formation in catalytic $\mathrm{CO}$ oxidation on Pt(110)," Science 292, 1357 (2001).

${ }^{9}$ M. Hildebrand, A. S. Mikhailov, and G. Ertl, "Nonequilibrium stationary microstructures in surface chemical reactions," Phys. Rev. E 58, 5483 (1998).

${ }^{10}$ Q. Tran-Cong-Miyata, S. Nishigami, T. Ito, S. Komatsu, and T. Norisuye, "Controlling the morphology of polymer blends using periodic irradiation," Nat. Mater. 3, 448 (2004).

${ }^{11} \mathrm{~J}$. O'Keefe and M. Recce, "Phase relationship between hippocampal place units and the EEG theta rhythm," Hippocampus 3, 317 (1993).

${ }^{12}$ Y. Yamaguchi, "A theory of hippocampal memory based on theta phase precession," Biol. Cybern. 89, 1 (2003).

${ }^{13} \mathrm{~S}$. Kondo and R. Asai, "A reaction-diffusion wave on the skin of the marine angelfish Pomacanthus," Nature (London) 376, 765 (1995).

${ }^{14}$ J. D. Murray, Mathematical Biology (Springer-Verlag, Berlin, 1989). The chapter for the model of the sea shell is on p. 505 .

${ }^{15} \mathrm{O}$. Steinbock, Á. Tóth, and K. Showalter, "Navigating complex labyrinths:
Optimal paths from chemical waves," Science 267, 868 (1995).

${ }^{16}$ T. Kohonen, Self-Organizing Maps (Springer, Berlin, 2001).

${ }^{17}$ I. Krastev and M. T. M. Koper, "Pattern formation during the electrodeposition of a silver-antimony alloy," Physica A 213, 199 (1995).

${ }^{18}$ Y. Nagamine and M. Hara, "Bifurcations of spatiotemporal stripe patterns formed by Ag/Sb co-electrodeposition," Physica A 327, 249 (2003).

${ }^{19}$ Y. Nagamine and M. Hara, "Surface-element analysis of spatiotemporal stripe patterns formed by Ag and Sb coelectrodeposition," Phys. Rev. E 72, 016201 (2005).

${ }^{20}$ Y. Nagamine and M. Hara, "Freezing of phase separation in spatiotemporal stripe patterns formed by $\mathrm{Ag}$ and Sb co-electrodeposition," Surf. Sci. 601, 803 (2007).

${ }^{21}$ Y. Nagamine and M. Hara (unpublished results). In this investigation, for the two patterns with one-directional anisotropic shapes (small wave structure and big wave structure) that emerged in the usual vertical electrode setup, dynamic behaviors were investigated. We found that the stripe width of the patterns does not change with time and stripes are annihilated when they collide. The characteristic properties are very similar to the patterns in a reaction-diffusion system such as the BZ reaction. This suggests that the two patterns with one-directional anisotropic shapes are formed by a reaction-diffusion system. However, the pattern shapes are anisotropic, unlike the BZ reaction which shows isotropic shapes. Therefore, the authors speculate that the shape anisotropy is derived from the convective flow.

${ }^{22}$ Physicochemical Hydrodynamics, edited by V. G. Levich (Prentice-Hall, Englewood Cliffs, NJ, 1962), p. 127.

${ }^{23}$ Application of Polarization Measurement in the Control of Metal Deposition, edited by I. H. Warren (Elsevier, Amsterdam, 1984), p. 253.

${ }^{24} \mathrm{X}$. Yang, S. Chen, C. Wang, and L. Li, "Effect of microenvironment on the potentiostatic-current oscillation of iron electrode in sulfuric acid solution,” Russ. J. Electrochem. 42, 491 (2006).

${ }^{25}$ D. Sazou, A. Diamantopoulou, and M. Pagitsas, "Conditions for the onset of current oscillations at the limiting current of the iron electrodissolution in sulfuric acid solutions," Russ. J. Electrochem. 36, 1072 (2000).

${ }^{26}$ See supplementary material at http://dx.doi.org/10.1063/1.3413962 for confirmation of the convective flow in setup B and no convective flow in setup C.

${ }^{27}$ Electron Microscopy in Solid State Physics, edited by H. Bethge and J. Heydenreich (Elsevier, Amsterdam, 1987), p. 244.

${ }^{28}$ Y. Nagamine, N. Kurono, and M. Hara, "Electric stability of spatiotemporal stripe patterns formed by silver and antimony co-electrodeposition under the constant current mode," Thin Solid Films 460, 87 (2004).

${ }^{29}$ Standard Potentials in Aqueous Solution, edited by A. J. Bard, R. Parsons, and J. Jordan (Dekker, New York, 1985).

${ }^{30}$ R. J. Field and R. M. Noyes, "Oscillations in chemical systems. V. Quantitative explanation of band migration in the Belousov-Zhabotinskii reaction," J. Am. Chem. Soc. 96, 2001 (1974).

${ }^{31} \mathrm{~K}$. Showalter, "Trigger waves in the acidic bromate oxidation of ferroin," J. Phys. Chem. 85, 440 (1981).

${ }^{32}$ J. M. Blakely, "Surface diffusion," Prog. Mater. Sci. 10, 395 (1963).

${ }^{33}$ R. A. Robinson and R. H. Stokes, Electrolyte Solutions (Butterworths, London, 1959), p. 513.

${ }^{34}$ P. Foerster, S. C. Müller, and B. Hess, "Critical size and curvature of wave formation in an excitable chemical medium," Proc. Natl. Acad. Sci. U.S.A. 86, 6831 (1989). 